\title{
Laser Processing by Using Diffractive Optical Laser Beam Shaping
}

\section{Technique}

\author{
Gediminas RAČIUKAITIS ${ }^{1}$, Evaldas STANKEVIČIUS ${ }^{1}$, Paulius GEČYS ${ }^{1}$, Mindaugas GEDVILAS ${ }^{1}$, Christian \\ BISCHOFF $^{2}$, Erwin JÄGER ${ }^{2}$, Udo UMHOFER ${ }^{2}$ and Friedemann VÖLKLEIN ${ }^{3}$ \\ ${ }^{1}$ Center for Physical Sciences and Technology, Savanoriu Ave. 231, LT-02300 Vilnius, Lithuania \\ E-mail: graciukaitis@ar.fi.lt \\ ${ }^{2}$ TOPAG Lasertechnik GmbH, Nieder-Ramstaedter-Str. 247, D-64285 Darmstadt, Germany \\ ${ }^{3}$ Institut für Mikrotechnologien, Hochschule RheinMain, Am Brückenweg 26, D-65428 Rüsselsheim
}

\begin{abstract}
Laser beam shaping and homogenization techniques are substantial to optimize a large number of laser-material processing applications and laser-material interaction studies. Diffractive optical elements (DOE) play important role in provision of the process-adapted laser beam shaping. In this paper, we present an approach for laser beam shaping by using innovative DOE. The circular Gaussian beam was transformed to a square flat-top intensity profile in the focal plane of the objective lens by using novel DOE from TOPAG Lasertechnik GmbH. About 95\% of the laser energy was coupled in the main beam with a nearly perfect rectangular shape. Experimental results have been achieved by applying the shaped beam for laser microfabrication. The picosecond and nanosecond lasers with moderate beam quality $\left(\mathrm{M}^{2}=1.5\right)$ were used in the experiment. The shaped laser beam was applied for direct laser ablation of metal film on the glass substrate, drilling in a silicon wafer, scribing of thin-film solar cells and other technical materials. This technique allows creating a rectangular-shaped flat-top intensity profile in the focal plane that shows distinct advantages in laser material processing.

DOI:10.2961/jlmn.2011.01.0009
\end{abstract}

Keywords: diffractive optics, beam shaper, laser processing, flat-top

\section{Introduction}

A Gaussian beam is a well-known and most convenient spatial laser beam distribution. It preserves its distribution when propagating and is excellent in focusing to a diffraction-limited spot. However, the spot area limited by a beam diameter $\left(1 / \mathrm{e}^{2}\right.$ level) contains only $86.5 \%$ of the laser beam power and intensity at the boundary is only $13.5 \%$ of the peak intensity. The energy in wings is lost or even causes several damage of surrounding material as well as a highintensity central peak. Because of threshold behavior of laser ablation the use of Gaussian beam profile is not favorable and most of laser material processing applications require uniform irradiation within a limited area with sharp edges on boundaries. This is especially true for thin-film structuring in electronics and photovoltaics, where high selectivity of the processing is top-most important. A uniform irradiance is further of interest for laser welding, laser micro-fabrication, laser radar, laser scanning and optical processing.

The laser beam with uniform intensity distribution over the cross-section of the working plane is called a flat-top beam. The problem of shaping a laser beam arose nearly together with invention of a laser. Frieden published the first paper on shaping of the laser beam to one with uniform irradiance in 1965 [1]. Beam shaping can be implemented by integration (averaging) and deterministic transformation. There exist several laser beam shaping techniques:

- Use of apertures when only the central flat portion of the beam is selected resulting in high energy losses [2];
- Multi-aperture beam integrators (homogenizers, microlens arrays, etc) [3];

- Field mapping transformation with refractive optics to get a uniformly distributed beam in the output plane, applicable to well-defined single mode laser beams [4]. Lossless transformations of the Gaussian beam to uniform irradiance use refractive, reflective or diffractive optics.

Refractive and reflective optical elements of a special shape are arranged usually in beam expander geometry. More intensive central part of the beam is spread over a larger area, while weak wings of the lateral beam distribution are concentrated closer to the center. Considerations about description of flat-top profiles are given in [5].

Transforming the beam irradiance profile (Gaussian to more uniform) by retaining the wave-front shape requires 2 beam shaping elements [6]. Examples of laser beam shaping optics are:

- refractive optical systems with at least two aspheric elements [7, 8];

- $\quad$ single bi-aspheric element [9];

- reflective optical systems [10];

- $\quad$ binary diffractive optics $[11,12,13,14,15]$;

- 1D beam shaping with adjustable phase slit [16].

A laser beam shaper consisting of two aspheric lenses provides a fixed geometrical radial remapping of the optical power entering the input aperture to the output aperture. It must be mounted and aligned carefully in order to use it most efficiently [8]. The shaper requires that the angular misalignment be less than about $140 \mu \mathrm{rad}$ with respect to 
the input beam, and translationally centered on the input beam to less than $25 \mu \mathrm{m}$. If the input laser beam has a poor profile, this will be echoed in the output beam profile. Only beams with a high quality $\left(\mathrm{M}^{2}<1.10\right)$ can give acceptable output profile flatness.

Flat-top intensity distribution can be realized through transformation of a single transverse mode Gaussian laser beam using an optical beam shaper. A low cost technique to fabricate the beam shaper that can convert a circular Gaussian beam into a circular flat-top beam was developed by [15]. The input beam size and laser wavelength should match its design specifications within $10 \%$ tolerances, and fine alignment between the input beam and the shaper within the $10 \mu \mathrm{m}$ tolerance is required. Flat-top size is altered by changing focal length of the objective. In general, an accurate continuous surface profile is hard to achieve by etching process, leading to performance degradation of the fabricated flat-top beam shapers [17]. Complicated beam shaping systems can afford very high shaping efficiency (95\% of energy in area of $90 \%$ intensity), however they require an ideal Gaussian beam and precise alignment. Sensitivity to misalignment and manufacturing inaccuracy can diminish and hide marginal improvement in performance from simple to advanced diffractive optics elements. Using simplified binary phase steps can greatly increase the beam shaper fabrication tolerance to low-cost flat-top beam shaper prototyping and production [15].

Processing, e.g. scribing thin films, with lasers with a circular Gaussian beam causes formation of non-smooth edges and damage or excess modification in overlapping areas $[18,19]$. A flat-top square-shaped beam is proposed to be ideal for laser processing. Use of a square-shaped laser beam prevented cracking of the thin films in a-Si solar cells $[20,21]$. A picosecond laser system with a special beam shaping optics was utilized for optimization of structuring processes of CIGS thin film solar cells [22]. Optimized top-hat optics with high straightness and steepness of the edges was developed using free-form refractive optics [23]. Processing results are demonstrated for various laser-based processes in thin film as well as crystalline $\mathrm{Si}$ solar cell production. The "free-shape" optics is difficult to fabricate [24] and that limits cost-effective application of the technology.

New diffractive optics technique called Fundamental Beam-mode Shaping (FBS) was applied to transformation of a circular Gaussian beam at the input plane of FBS into rectangular beam with nearly flat-top (top-hat) distribution at the focal plane of the focusing objective. FBS represents kind of a phase plate with a continuous and smooth phase relief profile to transform a beam to one of $\operatorname{sinc}(\mathrm{x})$-type in front of a focusing lens and which was realized by lithographic methods.

The FBS-shaped beam was applied in laser microfabrication experiments using picosecond and nanosecond lasers with moderate beam quality $\left(\mathrm{M}^{2}=1.5\right)$. The shaped laser beam was applied to direct laser ablation of metal film on the glass substrate, drilling in a silicon wafer, scribing of thin-film solar cells and other technical materials.

\section{Beam shaping technique}

The laser beam shaping is a process of redistributing the irradiance and phase. If a circular input Gaussian beam is focused by a lens, the intensity distribution in a focal plane of the lens remains circular Gaussian (Fig. 1). When a specially designed diffractive optical element for a flattop reshaping (FBS, for instance) is introduced in front of the lens, the intensity distribution in the focal plane can be transformed to a square-shaped beam (in lateral directions) with a flat-top intensity profile.
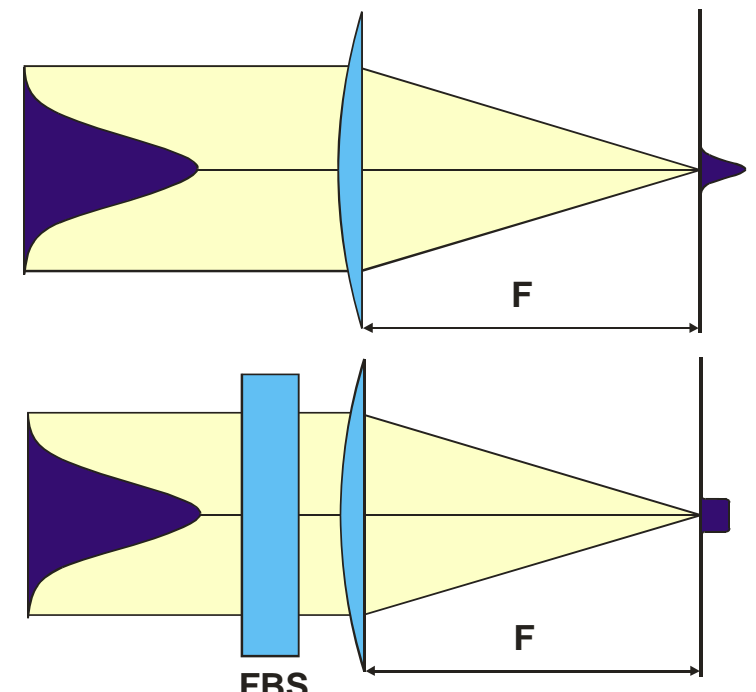

Fig. 1 Transformation of the beam profile by focusing with a lens without and with the FBS-type diffractive optical element in an optical path. Position of FBS along a beam path relative to the lens is not critical.

A diffractive optical element used in this research is a phase plate that converts the input Gaussian beam distribution into the top-hat beam after the objective lens. In the vicinity of the focal plane the beam forms spatial distribution with a flat-top and a square cross-section (Fig. 2). The FBS beam shaper represents kind of a phase plate with a continuous and smooth phase relief profile which was fabricated by lithographic methods.

a) Intensity [a.u.]

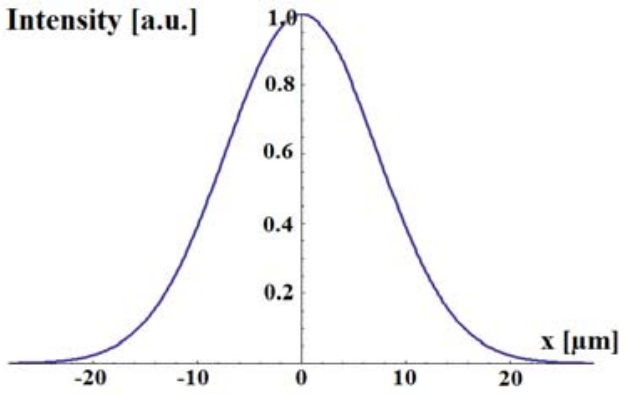

b)

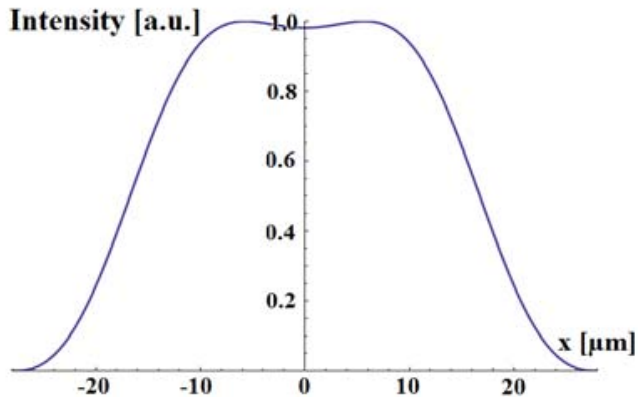

Fig. 2 Simulation of intensity distribution in a focal plane for a diffraction-limited Gaussian beam (a) and a beam trans- 
formed to a square top-hat with the FBS shaper (b) with $\lambda=532 \mathrm{~nm}$ and $\mathrm{NA}=0.011$

Efficiency of beam shaping from the circular Gaussian beam to the main top-hat profile is $95 \%$ in an area limited by $1 / \mathrm{e}^{2}(13.5 \%)$ level, and only $5 \%$ of energy is dissipated in wings. The flat-area uniformity of the top-hat profile is $\pm 2.5 \%$.

Characteristic of FBS is that the top-hat can be realized with a very small size that is near to the diffraction-limited spot. The minimum possible (top-hat) spot size is about 1.5-2 times that of the diffraction-limited Gaussian spot. Theoretically, steepness of the edges is diffraction-limited, but practically the limitations arose from quality of the objective and the initial beam $\left(\mathrm{M}^{2}\right)$. Depth of the top-hat focus is close to that for a Gaussian beam.

Size of the top-hat profile at $13 \%$ level $\left(1 / \mathrm{e}^{2}\right)$ can be adjusted by the focal lens and follows numerical aperture of the focusing objective according to equation [4]:

$$
2 \omega_{\text {top-hat }}=\frac{4 k \lambda}{\pi} \cdot \frac{F}{D} \approx \frac{\lambda}{N A},
$$

where $k$ is the empirical shaping parameter $k \sim 1.5 ; D$ is the diameter of the input beam on a lens; $F$ is the focal length of the objective. Numerical aperture of the objective affects only size but not a shape of the top-hat profile.

Specifications of the FBS beam shaper of TOPAG Lasertechnik $\mathrm{GmbH}$ are presented in Table 1. The shaper can be customized to transform an elliptical input beam.

Table 1 Specifications of the FBS top-hat beam shaper.

\begin{tabular}{ll}
\hline Material & Fused silica \\
Active area diameter & $2.0,2.5 \ldots 10.0 \mathrm{~mm}$ \\
Wavelength & $1064 \mathrm{~nm}, 532 \mathrm{~nm}, 355 \mathrm{~nm}$ or \\
& $266 \mathrm{~nm}$ (other on request) \\
Transmission & $>99 \%$ (with AR/AR coating) \\
Shaping efficiency & $>95 \%$ (in top-hat spot) \\
Uniformity & $+/-2.5 \%$ (of top-hat plateaus) \\
$\begin{array}{l}\text { Minimal lateral size } \\
\text { of top-hat spot }\end{array}$ & square, $\sim 1.5 \mathrm{x}$ of Gaussian spot \\
Installation & $\begin{array}{l}\text { In optics holder with x/y adjust- } \\
\text { ment }\end{array}$ \\
\hline
\end{tabular}

A profile of the well-prepared (spatial filtering to achieve $\mathrm{M}^{2}<1.1$ ) laser beam with the diameter of $5.5 \mathrm{~mm}$ shaped with the FBS element is shown in Fig. 3. The main part of laser energy is concentrated in the main top-hat peak and only weak wavelets detached from the main peak can be recognized.

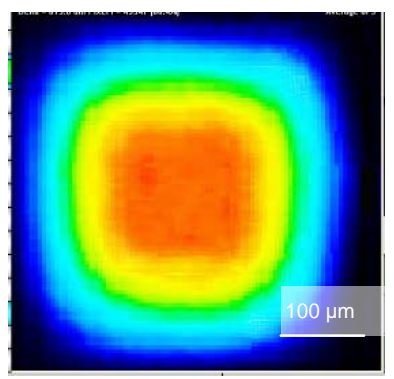

a)

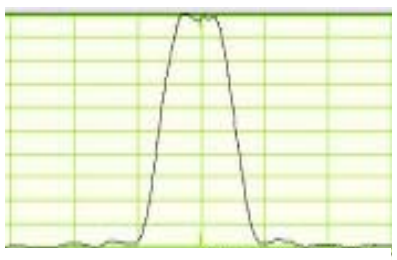

Scale $250 \mu \mathrm{m} / \mathrm{div}$
Fig. 3 Measured profile of the FBS shaped beam (a) and its cross-section (b): input Gaussian beam diameter $\left(1 / \mathrm{e}^{2}\right)$ $5.5 \mathrm{~mm}$, wavelength $1064 \mathrm{~nm}$, focusing lens $F=$ $1000 \mathrm{~mm}$.

The characteristics and specific advantages of the FBS top-hat beam shaper are:

- FBS beam shaper optics does not substitute the customer's focusing system (objective) but works together with the existing optics. FBS is a single thin optical element, which can be installed somewhere in the optical path before the focusing lens (easy installation);

- the flat-top beam profiles appear in the focal plane which simplifies the alignment of the optical setup;

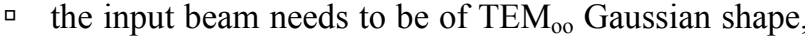
however the FBS beam shapers accept beams even with $\mathrm{M}^{2}=1.5$, as shown experimentally below;

- FBS shaper requires particular beam diameter, however, accepts a relatively high variation in the input beam diameter $(+/-5 \%)$.

- as a single thin optical element with simple and symmetric design, it is insensitive to angular misalignment, compared to remapping system [7-10];

a the depth of focus for the flat-top beam is close to Rayleigh length of the Gaussian beam focused with the same lens.

\section{Experimental}

\subsection{Evaluation of the beam shapers}

Experimental setup (Fig. 4) included a nanosecond laser NL15100 (Ekspla Ltd) working at $1064 \mathrm{~nm}$ with the maximum output power of $15 \mathrm{~W}$, attenuator made of $\lambda / 2$ plate and thin-film polarizer $\mathrm{P}$, a variable beam expander from Sill Optics to get the beam diameter of $3.8 \mathrm{~mm}$ (not shown in Fig.4), the FBS beam shaper, the focusing lens L with focal length of $500 \mathrm{~mm}$, two $4^{\circ}$ wedges $\mathrm{W}$ for reduction of the laser power and a CCD camera WinCamD with beam profile analyzing software (DataRay Inc). The laser was adjusted to generate a circular beam at pump level of $87 \%$, which corresponded to the mean output power of 12 W@100 kHz repetition rate. The laser power was controlled by an external attenuator. When the laser power was above $2 \mathrm{~W}$, neutral filters NF were installed in front of the CCD camera to prevent its overexposure.

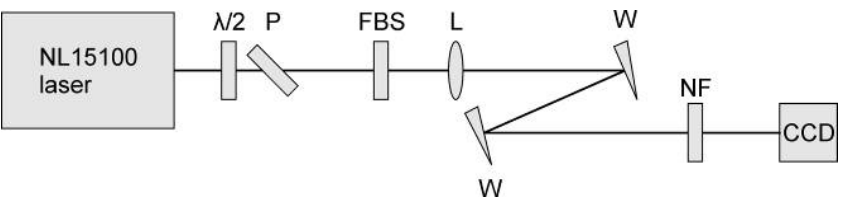

Fig. 4 Setup for investigation of the shaping performance of FBS with a high-power laser.

\subsection{Laser processing with shaped beams}

Processing experiments with a shaped nanosecond laser beam were performed with setup consisting of a laser NL640 (Ekspla), attenuator ( $\lambda / 2$ plate and thin-film polarizer P), beam shaping element FBS, beam guiding mirror $\mathrm{M}$ and a three-axis positioning system (Fig. 5). The focal length of an objective as well as the beam diameter were varied during the experiments.

Processing experiments with a shaped picosecond laser beam were performed with setup consisting of a high 
power, high repetition rate picosecond laser PL10100 (Ekspla) with external second harmonics module and galvoscanner HurryScan 14 (Scanlab) with a telecentric objective of a $80 \mathrm{~mm}$ focal length.

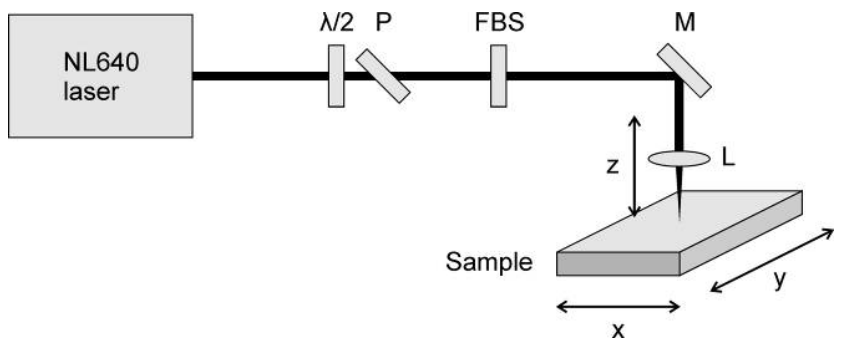

Fig. 5 Typical setup for laser ablation experiments using FBS.

All experiments were carried out in parallel with an unshaped close-to-Gaussian beam ("as-is") as well as with the FBS shaper installed in a beam path in front of the scanner. The beam diameter was optimized for the used FBS $\left(2 \mathrm{w}_{0}=\right.$ $5.5 \mathrm{~mm}$ ). Installation of FBS between experiments included its centering relative to the laser beam and was controlled by a CCD camera placed out of focus.

Table 2 Key parameters of the lasers used in experiments.

\begin{tabular}{lcccc}
\hline Laser & $\begin{array}{c}\text { Wave- } \\
\text { length, nm }\end{array}$ & $\begin{array}{c}\text { Max. output } \\
\text { power, W }\end{array}$ & $\begin{array}{c}\text { Pulse } \\
\text { duration }\end{array}$ & $\begin{array}{c}\text { Beam } \\
\text { quality, } \\
\text { M }^{2}\end{array}$ \\
\hline NL640 & 1064 & 6 & $10-18 \mathrm{~ns}$ & 1.3 \\
NL1500 & 1064 & 15 & $10-22 \mathrm{~ns}$ & 1.3 \\
PL10100 & 532 & 10 & $10 \mathrm{ps}$ & 1.5 \\
\hline
\end{tabular}

In laser ablation experiments with a shaped laser beam a chromium film (100 nm thick) on glass, a complete flexible multilayer structure of the CIGS solar cell ( $\mathrm{ZnO}$ topcontact $(1 \mu \mathrm{m}) ; \mathrm{CuIn}_{\mathrm{x}} \mathrm{Ga}_{(1-\mathrm{x})} \mathrm{Se}_{2}$ absorber layer $(2 \mu \mathrm{m})$; Mo back-contact $(1 \mu \mathrm{m})$ deposited on polyimide (PI) film $(25 \mu \mathrm{m}))$ were investigated.

The quality of processing was evaluated with an optical microscope (Olympus BX51) and a scanning electron microscope (SEM) JSM 6490LV (JEOL).

\section{Results and discussion}

\subsection{Shaping of high power laser beams with "imper- fect" beam profiles}

All the beam shaping techniques work well with ideal Gaussian beams $\left(\mathrm{M}^{2}<1.1\right)$. However, most of high power solid-state lasers have beam quality $\left(\mathrm{M}^{2}\right)$ in range of 1.31.5. Therefore, the main experimental task was to test novel FBS beam shapers with "real" high power and high repetition rate nanosecond and picosecond lasers, which are widely applied in modern technological processes.
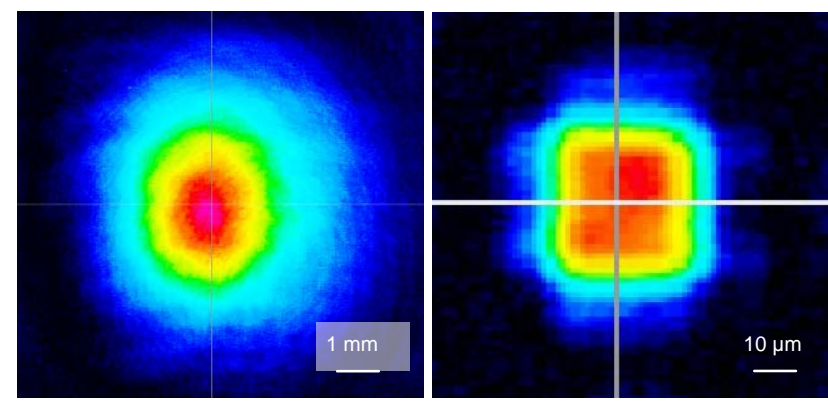

Fig. 6 Square-shaped flat-top laser beam intensity distribution (right) formed by using FBS and spherical lens and measured by using a CCD camera. Input beam profile is shown on the left. Mean laser output power $12 \mathrm{~W}$.

Fig. 6 shows beam profiles at output of the NL15100, laser and in the focal plane of objective when FBS was applied. The beam diameter after passing the beam expander was $3.8 \mathrm{~mm}\left(1 / \mathrm{e}^{2}\right.$ level) in both directions. The central square-shaped spot contained approximately $95 \%$ of laser power. Side-bands with $\sim 5 \%$ of laser power are out of the picture area. Asymmetry in the input beam profile did not disturb remarkably the shape of the main squareshaped spot and the intensity variation in the central flattop area was within $5 \%$.

The shape was monitored when the laser power was tuned by the external attenuator consisting of a-half-wave plate and thin-film polarizer to avoid any changes in laser operation and characteristics of the beam leaving the laser. High laser power up to $12 \mathrm{~W}$ did not exhibit any variations in the shaped beam profile.

\subsection{Ablation of thin metal films with the shaped top- hat beam}

Chromium films with the thickness of $100 \mathrm{~nm}$ were used to check the profile of the shaped beam and its ability to ablate the material. Experiments were performed with NL640 laser and setup, shown in Fig. 5. Squared openings were ablated in the metal film (Fig. 7).

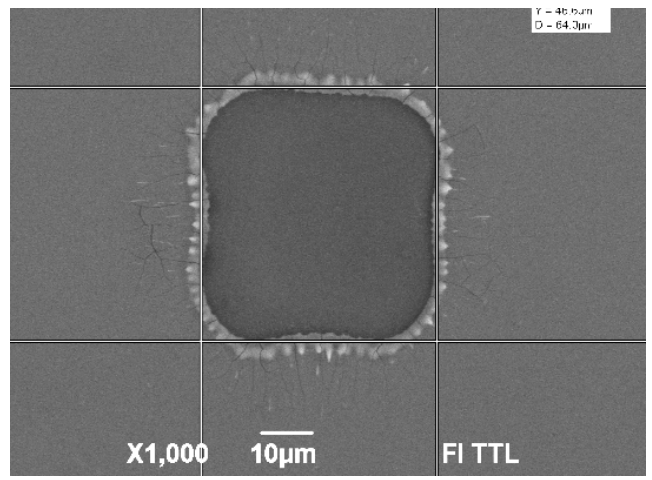

Fig. 7 SEM image of square-shaped hole ablated with a single laser pulse in a chromium thin film on the glass substrate using the FBS shaped beam. NL640 laser, pulse duration $6 \mathrm{~ns}$, wavelength $1064 \mathrm{~nm}$, Gaussian beam diameter@1/ $\mathrm{e}^{2}=3.8 \mathrm{~mm}$, beam quality $\mathrm{M}^{2} \sim 1.3$, focusing lens $F=50 \mathrm{~mm}$.

Size of holes depended on the used FBS shaper (dimension of active area) and the focusing lens. Features with a square shape as small as $20 \mu \mathrm{m}$ were ablated in the metal film using the $50 \mathrm{~mm}$ focusing lens and $5.5 \mathrm{~mm}$ FBS.

\subsection{Drilling of silicon wafers}




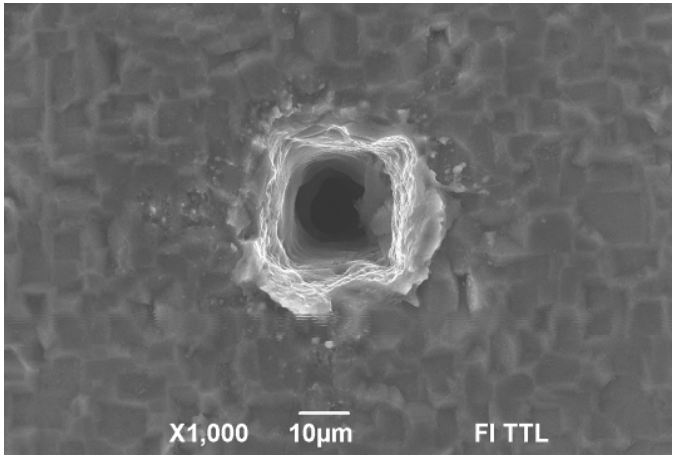

Fig. 8 Hole drilled in silicon wafer by percussion technique.

Experimental settings were the same as for metal film ablation: laser NL640, wavelength $1064 \mathrm{~nm}$, Gaussian beam diameter @ $1 / \mathrm{e}^{2}=5.5 \mathrm{~mm}$, focusing lens $\mathrm{f}=50 \mathrm{~mm}$. The objective position was fixed relative to the wafer. The resulting hole was of a rectangular shape up to the depth of about $100 \mu \mathrm{m}$ and passed into a circular shape deeper due to transformation of the beam profile during propagation out of focus (Fig.8).

\subsection{Scribing of thin-film CIGS solar cell with the shaped laser beam of a picosecond laser}

Laser scribing in multi-layered structures of modern thin-film solar cells requires high selectivity in ablation of the films without any effect on adjusted materials. A Gaussian beam profile does not permit achievement of distinct boundary, and use of flat-top shaped beams is proposed to be a solution [19]. Results of picosecond laser scribing of $\mathrm{ZnO}(1 \mu \mathrm{m}) / \mathrm{CIGS} / \mathrm{Mo} / \mathrm{PI}$ structure with Gaussian and tophat shaped beam profiles are shown below. Fig.9 presents results of laser scribing to expose a back contact of Mo (so called P3 scribe).

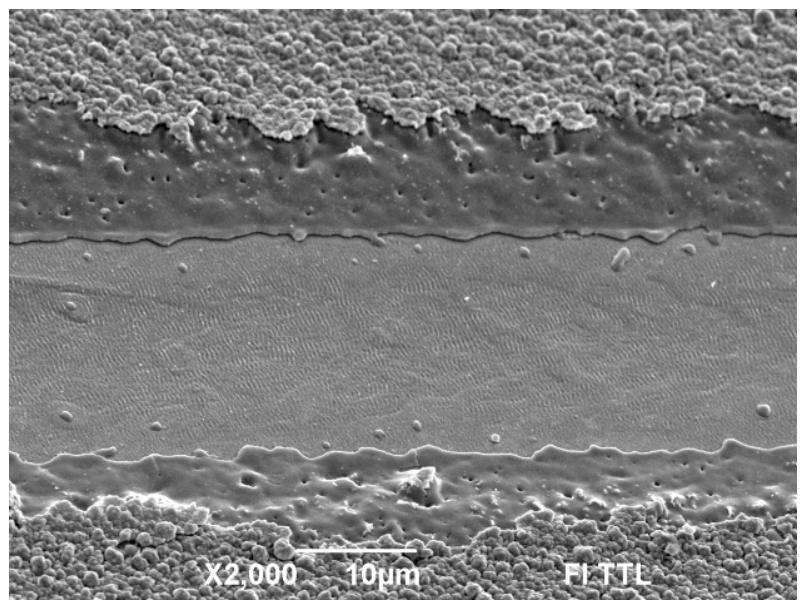

Gaussian profile beam

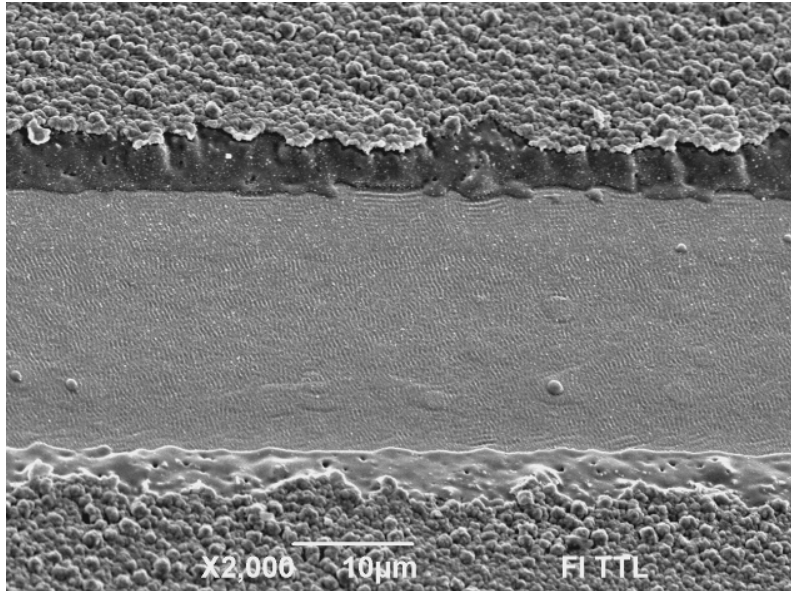

Top-hat profile beam

Fig. 9 Tilted SEM pictures of the $\mathrm{P} 3$ scribe in $\mathrm{ZnO}(1 \mu \mathrm{m}) / \mathrm{CIGS} /$ Mo/PI structure. Laser PL10100/SH, $370 \mathrm{~mW}, 100 \mathrm{kHz}$, $532 \mathrm{~nm}$; scanning speed $60 \mathrm{~mm} / \mathrm{s}$, single pass.

Wide areas of the disturbed absorber layer were left on both sides of the trench using the Gaussian beam with a smooth intensity profile. The FBS shaped beam had higher steepness of the beam edges, which caused reduction in a width of a modified area. The width of the trench was less sensitive to variation in the laser pulse energy for the FBS shaped beam (Fig. 10).

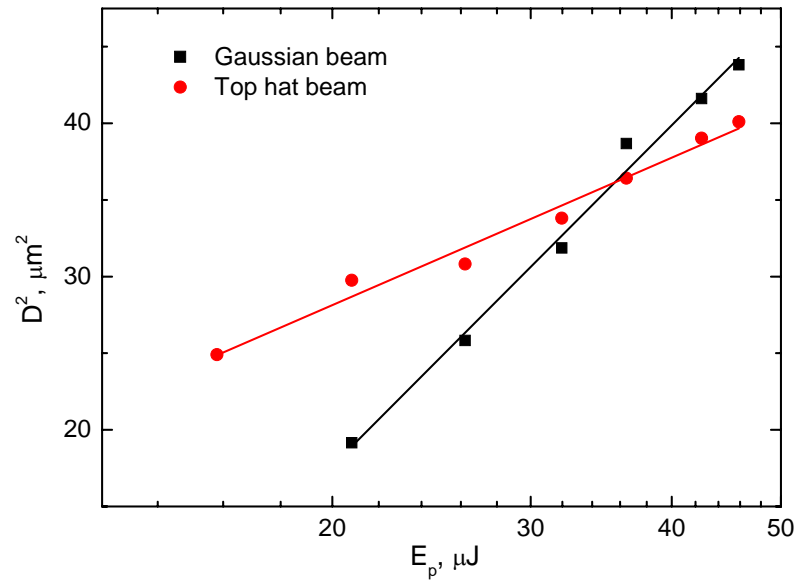

Fig. 10 Ablated spot diameter (Mo layer exposure - P3 process) vs. laser pulse energy. 10 pulse ablation. For the Gaussian beam: the Mo exposure threshold fluence $\mathrm{F}_{\text {th }}=$ $1.1 \mathrm{~J} / \mathrm{cm}^{2}$, beam waist $\mathrm{w}_{0}=32 \mu \mathrm{m}$.

Fig. 11 presents results of the top-contact removal with the picosecond laser. High speed scanning with a low beam spot overlap was applied. The circular Gaussian beam led to periodical modulated edge of the trench, while the square-shaped beam provided nearly a smooth edge along the scribe. 

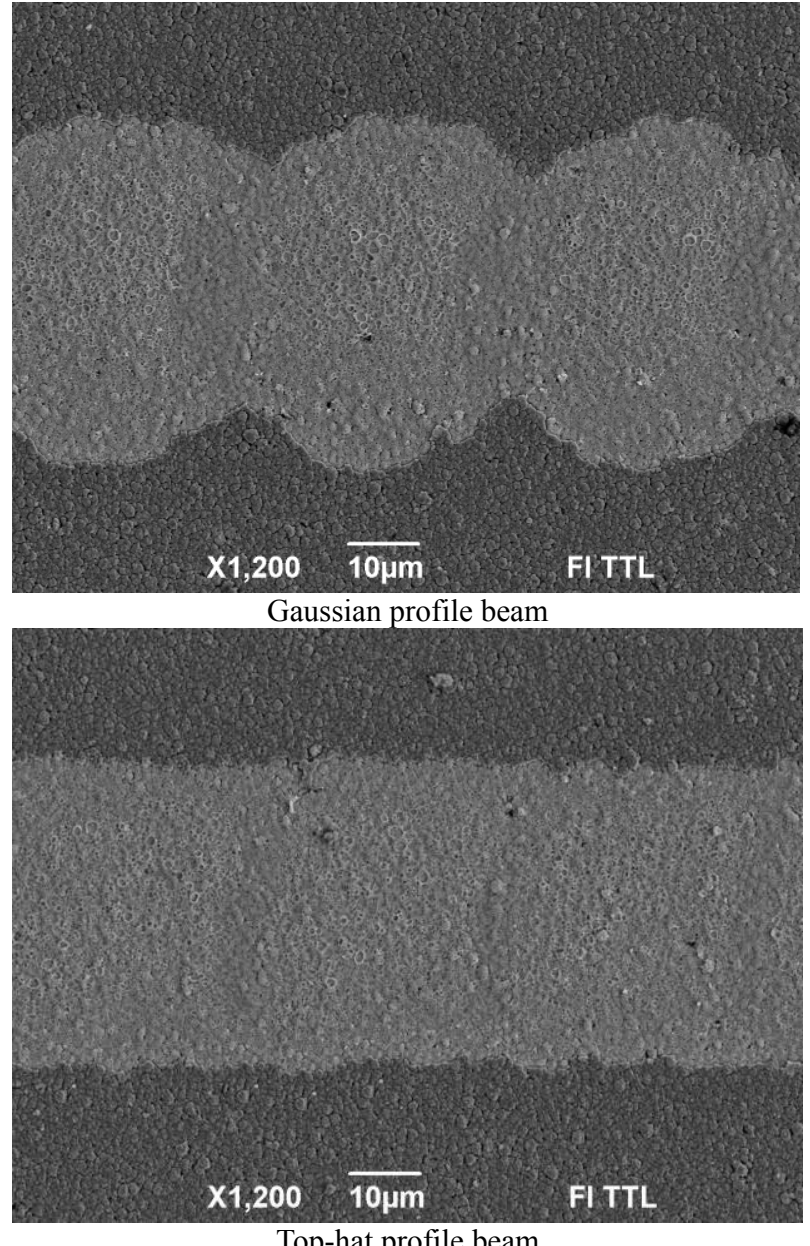

Top-hat profile beam

Fig. 11 Removal of a front contact in $\mathrm{ZnO}(1 \mu \mathrm{m}) / \mathrm{CIGS} / \mathrm{Mo} / \mathrm{PI}$ structure. Laser PL10100/SH, $370 \mathrm{~mW}, 100 \mathrm{kHz}$, $532 \mathrm{~nm}$; scanning speed $4.3 \mathrm{~m} / \mathrm{s}$, single pass.

\subsection{Ablation of silicon with the shaped laser beam of a picosecond laser}

The experimental setup with the picosecond laser was applied to ablate grooves in silicon wafer with Gaussian and top-hat shaped beams. Rectangular profile of the grooves was etched with the shaped beam and no recast material was found on its rims (Fig. 12).

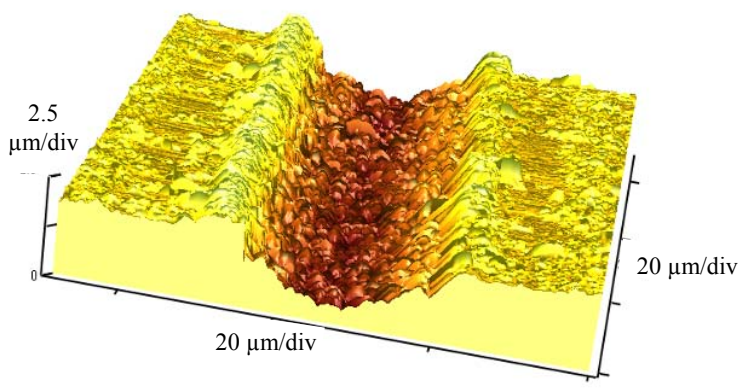

Gaussian profile beam

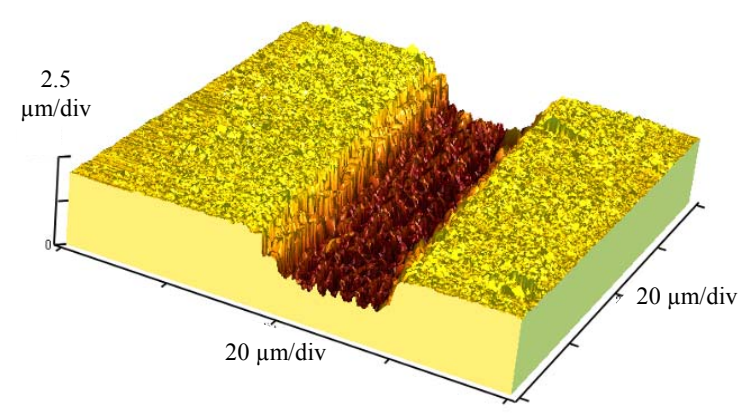

Top-hat profile beam

Fig. 12 Si ablation. Laser PL10100/SH, $225 \mathrm{~mW}, 100 \mathrm{kHz}$, $532 \mathrm{~nm}$; scanning speed $400 \mathrm{~mm} / \mathrm{s}, 40$ scans.

\section{Conclusions}

New DOE beam shaping optics, called FBS beam shapers, which deliver squared flat top beam profiles have been developed and applied for laser processing. For different applications such as scribing of thin films in solar cells, ablation of metal films and drilling in silicon, the material processing shows significant improvements compared to Gaussian beams. Scribing of the thin film layers with the Gaussian beam profile caused damage in the center of the laser-machined trench. To avoid damage and increase the process productivity it is necessary to convert it to a flat top profile beam. Furthermore, the square shaped flat top beam provided nearly smooth scribing edges and the width of scribing trenches was less sensitive to variation of pulse energy. The FBS beam shapers turned out to be relatively insensitive to variations of the input beam diameter of the Gaussian beam and also accept "imperfect" Gaussian beams with $\mathrm{M}^{2} \sim 1.5$. They deliver flat-top spots with the size of approximately $1.5 x$ larger than the diffraction-limited Gaussian spot. The flat-top spots lie in the focal plane of a focusing optics (objective), which simplifies the installation of the FBS beam shapers within existing optical setups.

\section{Acknowledgments}

DOE have been developed in the frame of an R\&D project (HA-Project-No.: 165/08-27) supported by Federal Government of Hessen (Germany) and Hessen Agentur, financed with LOEWE funds (Landes-Offensive zur Entwicklung Wissenschaftlich-ökonomischer Exzellenz, Förderlinie 3).

The work on laser structuring was partially supported by Research Council of Lithuania under project No AUT06/2010. We express our thanks to Dr. Š. Vaitekonis for AFM measurements.

\section{References}

[1] B.R.Frieden, Appl. Opt., 4, 1400-1403 (1965).

[2] F.M.Dickey, L.S.Weichman, R.N.Shagam, Proc. SPIE, 4065, 338-348 (2000).

[3] S. Bollanti, P.D. Lazzaro, D. Murra, Eur. Phys. J. Appl. Phys., 28, 179-186 (2004).

[4] F.M. Dickey, S.C. Holswade, Laser beam shaping: theory and techniques, Marcel Dekker, Inc. New York, Basel, 2000.

[5] Y. Li, Opt. Lett., 27, 1007-1009 (2002). 
[6] D.L. Shealy, in Frontiers in Optics, OSA Technical Digest (CD) (Optical Society of America, 2003), p. MOO1.

[7] P.W. Rhodes, D.L. Shealy, Appl. Opt., 19, 3545-3553 (1980).

[8] J.A. Hoffnagle, C.M. Jefferson, Appl. Opt., 39, 54885499 (2000).

[9] S. Zhang, G. Neil, M. Shinn, Opt. Express, 11, 19421948 (2003).

[10] P.H. Malyak, Appl. Opt., 31, 4377-4383 (19921).

[11] R. Saint-Denis, N. Passilly, M. Laroche, T.Mohammed-Brahim, K. Ait-Ameur, Appl. Opt., 45, 8136-8141 (2006).

[12] W.B. Veldkamp, C.J. Kastner, Appl. Opt., 21, 345356 (1982).

[13] W.B. Veldkamp, Appl. Opt., 21, 3209-3212 (1982).

[14] J. Cordingley, Appl. Opt., 32, 2538-2542 (1993).

[15] J.J. Yang, M.R. Wang, Opt. Eng., 42, 3106-3113 (2003).

[16] N. Passilly, M. Fromager, L. Mechin, C. Gunter, S. Eimer, T. Mohammed-Brahim, K. Ait-Ameur, Opt. Comm., 241, 465-473 (2004).

[17] Z. Liu, J.Y. Yang, M.R. Wang, in Frontiers in Optics, OSA Technical Digest (CD) (Optical Society of America, 2006), p. JWD12.

[18] P. Gečys, G. Račiukaitis, M. Ehrhardt, K. Zimmer, M. Gedvilas, Appl. Phys. A. (2010), 101, 373- 378 (2010).

[19] G. Račiukaitis, P. Gečys, J. Laser Micro/ Nanoeng., 5, 10-15 (2010).

[20] S. Haas, A. Gordijn and H. Stiebig, Prog. Photovolt: Res. Appl., 16, 195 (2008).

[21] S. Haas, G. Schope, C. Zahren, H. Stiebig, Appl. Phys. A., 92, 755-759 (2008).

[22] E. Steiger, M. Scharnagl, M. Kemnitzer, A. Laskin, Proc. of 28th Int. Congress on Applications of Lasers \& Electro-Optics (ICALEO 2009), p. M1107.

[23] O. Homburg, B. Braun, U. Jager, F. Lottspeich, A. Grohe, T. Mitra, Proc. of 28th Int. Congress on Applications of Lasers \& Electro-Optics (ICALEO 2009), p. M1109.

[24] R. Kuwano, T. Tokunaga, Y. Otani, N. Umeda, Opt. Review, 12, 476-479 (2005). 